\title{
IL ROMANTICISMO IN ITALIA
}

\author{
Luigi Castagnola \\ Universidade do Paraná
}

SOMMARIO: 1. Romanticismo storico e romanticismo psicologico. 2. L'origine del romanticismo storico ha luogo fuori d'Italia. - 3. Nascita e trionfo del romanticismo in Italia. 4. Secondo e terzo romanticismo italiano. - 5. Aspetti negativi e positivi del romanticismo italiano.

1. - Romanticismo storico e romanticismo psicologico.

Quando gli studiosi e gli storici di una lingua - qualunque essa sia - vogliono cimentarsi a fissare, puntigliosamente, il suo anno di nascita, o pretendono decretare, funereamente, quello della sua morte, ci dànno l'impressione d'essere nervosi, incerti, stizziti, imbarazzati. Ed è inevitabile che sia così. In realità, una lingua non potrà mai avere il suo anno di nascita, nè vedrà spuntare, felicemente, quello della sua morte. Atto di nascita e atto di morte non esistono per questa fenice che drizza eternamente le sue penne dorate verso i lidi di una vita perenne quanto l'umanità.

Non è, quindi, un paradosso asserire che una lingua non muore mai (1). Potrà morire, in certo senso, la lingua di questo o di quell'altro scrittore, ma la lingua del popolo non si spegne nè s'interrompe. Essa continua la sua vita modificandosi, trasformandosi, rinnovandosi sempre in nuove forme,

1) Così un linguista potè scrivere che la lingua italiana, francese, spagnola, portoghese, romena si possono considerare come "la stessa lingua latina quale si parla a distanza di secoll in Italia, in Francia, in Spagna, in Portogallo, in Romania". Cf. Salvatore BATTAGLIA, Introduxione alla Linguistica Romanza, Piront1, Napoli, 1946, p. 7. 
sotto la spinta di numerosi e complessi fattori storici, linguistici, attraverso la parlata viva, fluida, cangiante, mobilissima del popolo. Non si possono concepire popoli senza parola, muti come pesci.

Gli scrittori ed i poeti calano in questa corrente mobile di espressione i loro libri ed i loro poemi, che manifestano i pensieri, i sentimenti, le passioni, le gesta, il progresso degli individui e delle nazioni. E, dato che la natura dell'uomo è fondamentalmente la stessa in tutti i tempi ed in tutti i luoghi, si ripetono certi temi dello spirito nel corso storico delle letterature. Uno di questi temi è certamente il romanticismo psicologico.

Il romanticismo è, senza dubbio, uno dei grandi momenti culturali e artistici delle grandi nazioni europee. Storicamente esso ebbe la sua comparsa aurorale sullo smorire del secolo XVIII e la sua esplosione trionfante nel secolo XIX. E fu un movimento così vasto e complesso che improntò di sè tutta un'epoca, precisamente l'epoca romantica. Lasciando da parte ogni altro aspetto del romanticismo - per quanto è possibile - , consideriamo qui soltanto quello letterario.

Davanti alla vastità del fenomeno romantico, più d'uno ha esitato a darne la definizione e ci fu perfino chi, disperato dalla difficoltà dell'impresa, sostenne la sua indefinibilità (2). Sulla questione è ritornato, recentemente, Nicola Petruzzellis in un suo libro, che dimostra fine acume critico e vasta competenza filosofica (3), e dove si contesta l' opinione della pretesa impossibilità di definire il romanticismo. "Le grandi epoche della cultura attraggono tanto più vivo interesse quanto più difficile appare la loro sintesi interpretativa, onde il moltiplicarsi delle definizioni, delle ipotesi e dei tentativi erme-

2) Cf. R. BOTTACCHIARI, La Rivoluzione romantica, Roma, 1943, p. 5. Tale opinione manifestò pure T. DICKINSON: "O têrmo "romantismo" abrange tal variedade de sentidos - social, político, psicológico, assim como literário - que não é possível dar-lhe definição exata". Cf. Thomas H. DICKINSON, História da Literatura Norte-Americana (trad. port.), S. Paulo, IPE, 1948, p. 149.

3) Cf. N. PETRUZZELLIS, L'Estetica dell'idealismo, Cedam, Padova, 1950. 
neutici, che, quando si fondano su nutrita cultura, lungo studio e grande amore, giovano sempre al progresso degli studi, ancorchè talvolta pecchino in prospettiva mettendo in primo piano questo o quell'altro aspetto unilaterale, frettolosamente scambiato per l'essenza del fenomeno storico che si voleva studiare. Così è avvenuto che anche sul Romanticismo venisse crescendo una lussureggiante letteratura critica, che, pur nella frequente unilateralità di giudizi, è riuscita almeno a chiarirne gli espetti diversi. Ma a velarne l'ispirazione profonda nell' arte e nel pensiero hanno contribuito pregiudizi di varia natura e origine, come quello della sua pretesa indefinibilità e irriducibilità a un concetto e a un concetto univoco, data anche la varietà dei suoi aspetti, nonchè l'esasperata ricerca dei precursori" (4).

Non mancarono tentativi, da parte italiana, di far derivare il romanticismo, compreso quello tedesco, dal barocco; si vollero perfino vedere precedenti romantici nella sentimentalità del Guarini e del Tasso, e qualcuno ebbe l'ardire di riscontrare già uno pseudo-romanticismo "nell'accorata nostalgia di Mimnermo o nella dolce malinconia catulliana" (5), sebbene tali teorie non abbiano mai potuto essere prese sul serio.

Una delle cause che sembrano rendere impossibile la definizione del romanticismo si deve riporre, se non erriamo, nella mancanza di distinzone tra l'aspetto storico e quello psicologico del romanticismo. Gli scrittori, ed in particolare i poeti, portano connaturate in sè molte di quelle caratteristiche e tendenze spirituali che i romantici predilessero e sventolarono come bandiera. I letterati, i poeti, gli artisti sono sempre un po' fantastici, sognatori, chimerici, agitati da passioni mal dominate o spavaldamente sbrigliate, ansiosi di concretare in nuove forme i fantasmi che urgono con forza nella loro immaginativa, idealisti che vanno in cerca di bellezze nuove, come i nobili cavalieri antichi andavano in traccia della

4) N. PETRUZZELLIS, op. cit., pp. 13-14.

5) N. PETRUZZELLIS, op. cit., p. 16. 
dama ideale. Ed è raro che anche in prosatori notoriamente vigilanti contro le improvvise bizzarrie della fantasia - la pazza di casa - e guardinghi contro le morbose eruzioni del sentimento, non si trovino pagine di scomposta passione, ventate di torbida nostalgia, accorati accenti di lacrimante tristezza, scatti guerreschi alla "Sturm und Drang", languenti notti lunari in cui lo spirito sogna veleggiando verso plaghe cosmiche indefinite. Sono questi tutti aspetti e altrettanti te$\mathrm{mi}$ dell'eterno romanticismo psicologico, che pullulano con vicenda alterna tanto nel mondo dei poeti classici come in quello dei bardi romantici.

Ecco perchè la critica, nitidamente oggettiva, coglie spunti prettamente romantici nelle opere dei più puri paladini del classicismo. Prendiamo, ad esempio, un poeta e prosatore ritenuto un araldo della scuola classicista: Ugo Foscolo (6). Tutti gli storici della letteratura italiana hanno scoperto nella produzione foscoliana, tanto poetica che prosastica, profondi vestigi di un acceso spirito romantico. Per dimostrare quanto affermiamo, citeremo due brani del cantore de "Le Grazie"; uno in poesia, l' altro in prosa.

Dal carme "I Sepolcri":

$$
\text { e forse l'ossa }
$$

Col mozzo capo gl'insanguina il ladro

Che lasciò sul patibolo i delitti.

Senti raspar fra le macèrie e $i$ bronchi

La derelitta cagna ramingando

Su le fosse e famèlica ululando;

E uscir dal teschio, ove fuggìa la Luna,

L'ùpupa, e svolazzar su per le croci

6) Parlando dell'arte foscoliana A. Pompeati scrisse: “...dall'Ortis sale aj Sepolcri e alle Grazie, cioè dalla vampata del più impetuoso romanticismo alla contemplazione ferma e consapevole della bellezza". Cf. A. POMPEATI,Storia della Letteratura Italiana, UTET, Torino, 1953, vol. IV. p. 35 
Sparse per la funèrëa campagna,

E l'immonda accusar col luttüoso

Singulto i rai di che son pie le stelle

Alle obliate sepolture. (7)

Il Foscolo parla del suo grande amico e maestro, Parini, con la musa Talia, e con essa si lamenta perchè Milano non consacrò al suo vate nè un'urna, nè una modesta lapide, ma permise che il famoso cantore del "Giorno" fosse sepolto promiscuamente nel cimitero comune, dove si sepellivano anche i giustiziati. Il poeta dei "Sepolcri", in compagnia della musa Talia, ispiratrice della poesia satirica pariniana, s'affanna nella ricerca notturna, in cimitero, della tomba dove dorme "il sacro capo" del suo amico. E nell'accesa fantasia crea la lugubre scena cimiteriale di un colorito ossianesco e romantico: vede il mozzo capo del ladro, che finì sul patibolo la sua vita delittuosa, lordare di sangue le spoglie del Parini; vede la cagna derelitta aggirarsi famelica fra le ossa dei morti e raspare fra i sepolcri diruti, ululando, per trovare sparsi resti mortali da rosicchiare; vede l'upupa uscire dal suo macabro nascondiglio - il teschio di qualche morto - e svolazzare con volo immondo su per le croci del cimitero, lanciando il suo lugubre strido desideroso di una tenebra completa senza stelle.

Dalle "Ultime lettere di Jacopo Ortis":

"Sono salito su la più alta montagna: i vènti imperversavano; io vedeva le querce ondeggiar sotto a miei piedi; la selva fremeva come mar burrascoso, e la valle ne rimbombava; su le rupi dell'érta sedeano le nuvole - nella terribile maestà della natura la mia anima attònita e sbalordita ha dimenticato i suoi mali, ed è tornata per alcun poco in pace con me medesimo.

Vorrei dirti di grandi cose: mi passano per la mente; vi sto pensando! m'ingombrano il cuore, s'affollano, si confondono; non so più da quale io mi debba incominciare: poi tutto ad un tratto mi sfuggono, ed io prorompo in un pianto dirótto.

7) Ugo FOScOLO, Dei sepoleri, vv. $75-86$. 
Vado correndo come un pazzo senza saper dove e perchè: non m'accorgo, e i miei piedi mi trascinano fra' precipizi...

... I nostri occhi morenti chiedono altrui qualche stilla di pianto, e il nostro cuore ama che il recente cadavere sia sostenuto da braccia amorose, e cerca un petto dove trasfondere l'ultimo nostro respiro. Geme la natura perfin nella tomba, $\mathrm{e}$ il suo gèmito vince il silenzio e l'oscurità della morte.

M'affaccio al balcone ora che la immensa luce del sole si va spegnendo, e le tenebre rapiscono all'universo que' raggi languidi che balenano su l'orizzonte; e nella opacità del mondo malinconico e taciturno contemplo la immagine della Distruzione divoratrice di tutte le cose. Poi giro gli occhi sulle macchie de' pini piantati dal padre mio su quel colle presso la porta della parrocchia, e travedo biancheggiare fra le fronde agitate da' venti la pietra della mia fossa. Quivi ti vedo venir con mia madre, e pregar pace non foss'altro alle ceneri dell'infelice figliuolo. Allora dico a me stesso: Forse Teresa verrà solitaria sull'alba a rattristarsi dolcemente su le mie antiche memorie, e a dirmi un altro addio. No! la morte non è dolorosa. Che se taluno metterà le mani nella mia sepoltura e scompiglierà il mio scheletro per trarre dalla notte in cui giaceranno le mie ardenti passioni, le mie opinioni, i miei delitti - forse, non mi difendere, Lorenzo, rispondi soltanto: Era uomo, e infelice" (8).

In questo brano non si vede solamente un'arte ancora imperfetta e una forma di sapore ricercato e arcaicizzante, ma l'espressione di sentimenti coloriti d'un languore che più tardi - come fu bene osservato - si sarebbe chiamato romantico.

Altra poetica, altro spirito aleggia nella lucente e chiara ode "A Luigia Pallavicini caduta da cavallo". Eccone alcune strofe:

\section{I bàlsami beati}

Per te le Grazie apprestino,

Per te i lini odorati

8) Ugo Foscolo, Ultime lettere di Jacopo Ortis, dalla lettera del 23 maggio. 
Che a Citerèa porgeano

Quando profano spino

Le punse il piè divino, Quel dì che insana empiea

Il sacro Ida di gemiti

E col crine tergea

E bagnava di lagrime

Il sanguinoso petto

Al cìprio Giovinetto.

E te chiama la danza

Ove l'aure portavano

Insòlita fragranza,

Allor che, a' nodi indòcile,

La chioma al roseo braccio

Ti fu gentile impaccio:

Tal nel lavàcro immersa,

Che fiori, dall'inachio

Clivo cadendo, versa,

Palla i dall'elmo liberi

Crin su la man che gronda

Contien fuori dell'onda.

Armoniosi accenti

Dal tuo labbro volavano,

E dagli occhi ridenti

Tralucéano di Venere

I disdegni e le paci,

La speme, il pianto, e i baci. (9)

Qui siamo in pieno classicismo. Sembrano versi di un altro lirico, di un poeta che vive nell'edonistico mondo mitologico della serena Ellade, popolata di dee.

Tali contrasti si potrebbero facilmente incontrare in quasi tutti i poeti, tanto classici che romantici. Dimostrò, quindi,

9) Ugo Foscolo, A Luigia Pallavicini caduta da cavallo, vv. $1-12$ e $19-36$. 
un acuto senso critico Arturo Pompeati quando scrisse nella sua monumentale "Storia della Letteratura Italiana" (10) che una delle difficoltà che si oppongono ad una definizione del romanticismo "nasce dal fatto che accanto all'aspetto storico del romanticismo" ci si presenta "quello psicologico", che non conosce limiti di tempo e di spazio. "Quando in uno scrittore prevale il sentimento al punto di forzare i controlli della ragione; quando alla visione del concreto si sovrappone l'ebbrezza del fantastico e del chimerico; quando l'osservazione della realtà suscita nello scrittore contrasti e passioni che lo spingono alla negazione o alla fuga; quando all'amore delle forme nette, limpide, rilevate si sostituisce la predilezione per la parola accesa, enfatica, spavalda, oppure per le linee sfumate, trepide, atte più a suggerire che a esprimere; quando l'io di chi scrive si esibisce con un'ostentazione clamorosa e ingombrante, allora siamo in presenza di manifestazioni romantiche, anche se estranee alle fortune storiche della scuola romantica. Perciò, al modo stesso che casi sporadici di temperamento romantico si possono cogliere in età di imperante gusto classico, ci accade di ravvisare nella formazione di scrittori maturatisi poi in un classicismo impeccabile momenti di inquietudine, o perfino di disordine romantico" (11).

Per queste ragioni precisamente, gli storici della letteratura italiana sentono la difficoltà di "accordare alla costrizione della scuola (romantica) la libera presenza dei due grandissimi spiriti di quella generazione: il Manzoni e il Leopardi" (12). Il Manzoni è l'esponente massimo del romanticismo italiano; eppure nessuno oserebbe, parlando del grande prosatore e poeta lombardo, ricordare le severe parole del Goethe: "Classico chiamo ciò che è sano; romantico ciò che è malato". Ed a proposito del Leopardi Attilio Momigliano, critico ed esteta, potè parlare in questi termini: "Il rinnovato classicismo è il fenomeno letterario dominante del periodo fra l' Arcadia e il

10) Cf. Arturo POMPEATI, Storia della Letteratura Italiana, UTET, Torino, vol. IV, p. 34 .

11) A. POMPEATI, op. cit., pp. 34-35.

12) A. POMPEATI, op. cit., p. 35. 
romanticismo: da quella scuola vien fuori Giacomo Leopardi. La sua cultura si forma in un momento di fervore filologico e mentre i dittatori del gusto sono il Monti e il Giordani; le sue affermazioni teoriche sono per il classicismo e contro il romanticismo... Egli sembra il più classico dei nostri lirici per il dominio che esercita su' suoi sentimenti, per l'aspetto lineare con cui si presentano le sue liriche, per la purezza immobile della sua forma" (13).

Tutto ciò dimostra che anche i migliori storici ed i critici più scaltri scoprono senza difficoltà profonde tracce di romanticismo psicologico in autori che fiorirono nel periodo storico d'imperante classicismo e pagine di puro e genuino sapore classico in autori che sono considerati i più legittimi rappresentanti del romanticismo storico.

Curiosa è del resto la storia della parola "romantico". Secondo L. P. Smith, essa nacque in Inghilterra verso la fine del secolo XVII, e significava "francese", specialmente "francese antico". Infatti è noto che agli albori della letteratura francese, in lingua d'oil, apparvero i famosi "romans" del ciclo brettone: Roman de Brut, o Geste des Bretons. E anche nel ciclo classico della letteratura francese di quell'epoca abbondarono i "romanzi": Roman de Troie, Roman de Thèbes, Roman d'Éneas, Roman de Jules César, Roman d'Hector, ecc. $\mathrm{Nel}$ secolo XIII fu elaborato il vasto romanzo in versi - quasi quattromila ottonari -, intitolato Roman de la rose; famoso è pure il Roman de Renard, prolisso poema che narra le avventure della volpe e del lupo.

Se, da principio, la parola "roman" servi a designare una narrazione in versi" (14), quando la poesia epica e cavalleresca decadde, "servi anche a indicare il racconto in prosa, cioè il romanzo. E romantic venne a esprimere cosa da romanzo, cioè diversa dalla realtà consueta" (15). Nel secolo XVIII un

13. Attilio MOMigliano, Storia della Letteratura Italiana, Principato, Milano, 1950, p. 428.

14) A. POMPEATI, op. cit., p 36.

15) Idem, ibidem, p. 36. 
vago sentimentalismo invase la vita e la letteratura, e la parola "romantico" fu usata "per esprimere uno stato d'animo tenero e sognante o anche un paesaggio selvaggio o grandioso" (16). Nello stesso secolo, il razionalismo filosofico chiamò "romantic ogni eccesso del sentimento, ogni aberrazione irragionevole. Sopraggiunge poi il gusto del medioevo, del "gotico", e l'Inghilterra è la prima ad esserne presa, e battezza romantico il gotico, appunto, il medievale, contrapposto a quello che è classico e antico" (17).

La Germania, dopo essersi impossessata della parola, vi costrui attorno la teoria di una nuova forma di cultura e di arte, che sarà appunto il romanticismo. Herder svelò il grande valore estetico che stava racchiuso nella poesia popolare del Medio Evo e mise in luce l'importanza nazionale di quella poesia per il popolo germanico. Novalis - il più famoso poeta del romanticismo tedesco — "adottò il termine" romantik" per indicare le nuove esigenze spirituali ed estetiche e il carattere della scuola letteraria che le esprimeva" (18).

Da questa lunga vicenda a cui fu sottoposta la parola "romantico", si vede quanto fu lunga e fluttuante la storia del suo significato. Il vocabolo, che per la sua radice ci porta col pensiero a Roma classica e antica, finì, dopo tanta evoluzione, per indicare una tendenza spirituale ed artistica antiromana.

La poetica del romanticismo, dunque, se appartiene, per il suo aspetto storico, ad un periodo determinato della cultura artistica europea, per il suo aspetto psicologico s'incontra in epoche lontane dal romanticismo.

2. - L'origine del romanticismo storico ha luogo fuori d'Italia.

Guardando le cose senza gli occhiali del campanilismo, sembra di notare fra le nazioni e le culture europee una specie di mutuo scambio spirituale ed artistico. Pare quasi che ogni popolo di quel continente si sforzi per retribuire le offerte cul- 
turali ricevute dagli altri popoli, rinviando loro il ricco e nuovo patrimonio di un proprio mondo artistico, creato dall'intelligenza autoctona. In tal modo si determina, attraverso i secoli, nella storia della cultura europea "un circolo di anime alterne" - secondo la fortunata espressione di uno studioso che ha per risultante un sempre maggiore arricchimento spirituale di quelle operose civiltà.

Infatti, la cultura greco-romana classica alimentò il sapere e l'arte d'Europa durante lunghi secoli, ed in certo senso essa continua, in un cerchio più vasto, ad informare la cosiddetta cultura occidentale; la Francia gotica, medievale e cavalleresca andò, a sua volta, alla conquista delle nazioni, incontrando ovunque accogliente ospitalità e cittadinanza; l'Italia rinascimentale crea un nuovo ciclo di cultura artistica - il Rinascimento - che dalla penisola mediterranea si estende fino alle isole britanniche; la Germania, finalmente, invia il suo messaggio romantico accolto dovunque con entusiasmo. Nessun popolo riceve passivamente i doni dello straniero, ma li assimila dando loro un'impronta propria, e li adatta alle esigenze storiche e tradizionali della sua gente. Sicchè, per esempio, il romanticismo germanico, inglese, francese, italiano saranno movimenti somiglianti, ma differenziati da caretteristiche particolari ben evidenti a noi osservatori postumi, e non intieramente sconosciute ai loro protagonisti.

Il romanticismo storico, come è noto, è "un movimento di pensiero, di spirito, che sorse in netta reazione al Razionalismo, all'Illuminismo, al Cosmopolitismo rivoluzionario. Massime care al Romanticismo furono: all'infuori dell'universalismo cattolico non si possono ammettere altri universalismi; ogni popolo ha una patria e quindi una sua storia; i popoli per non decadere o per rinnovarsi devono risalire alle loro tradizioni, alla loro storia; la scienza non è fine a se stessa, ma ha un compito educativo" (19).

19) Giovanni SORANZO, Avviamento agli Studi Storici, Mazorati, Milano, 1950, p. 233. 
La patria di origine del Romanticismo è la Germania. Qui il movimento romantico ebbe la sua preparazione, la sua struttura dottrinaria, i suoi primi grandi poeti. Dalla Germania emigrò verso il nord (Inghilterra), verso ovest (Francia) e verso il sud (Italia); prese pure altre direzioni e il suo volo arrivò anche in terre lontane, come le steppe nevose del Caucaso e i lidi affascinanti del meraviglioso mondo americano.

Nella patria di Arminio si deve considerare come una preparazione al romanticismo l'opera di quella tempestosa schiera letteraria che agitò la bandiera dello Sturm und Drang. Vi parteciparono quasi tutti i grandi appassionati della letteratura che vissero in quell'epoca e, per qualche tempo, anche Schiller e Goethe. Gli adepti a questa scuola concepivano la vita come istinto spontaneo, ingigantimento dell'esistenza che ognuno doveva conquistare nella più assoluta libertà da ogni legge tradizionale. Nè la vita, nè l'arte dovevano più ubbidire alle norme antiche, ma tutto si doveva ricostruire in base all'innata disciplina della stirpe. L'arte doveva ricercare la bellezza non in forme statiche e definitive, ma nella dinamicità dello slancio e dell'impulso istintivo. Lo spirito era tentato dal desiderio di dar vita a una estetica nuova. Con questi principi e con questa orientazione lo Sturm und Drang preparò gli animi per l'imminente nascita del romanticismo.

Anche Schiller e Goethe - sebbene giganti solitari il cui alto volo li stacca da ogni scuola - ebbero la loro parte in quell'aurora piena di promesse che preannunziava la nuova scuola. Ecco quel che Eckermann fa dire a Goethe in una conversazione amichevole: "Il concetto della poesia classica e romantica, che ora è diffuso in tutto il mondo e cagiona tanti contrasti e disordini, è partito da me e da Schiller. Io seguo, nella poesia, per norma, il procedimento obiettivo, e volli far prevalere solamente questo. Ma Schiller, che procedeva in tutto subiettivamente, considerò la sua maniera come la giusta, e per difendersi contra di me, scrisse la memoria 
sulla poesia ingenua e sentimentale. Egli dimostrò che io stesso sono un romantico, e che la mia Ifigenia, per il predominio del sentimento, non è affatto così classica e della maniera antica, como altri potrebbero credere. Gli Schlegel s'impadronirono dell'idea, la lanciarono così che essa è diffusa ora per tutto il mondo, e ciascuno parla di classicismo e di romanticismo; cosa a cui cinquant'anni fa non pensava nessuno" (20).

Il 1797 è preso come data ufficiale per segnare, convenzionalmente, la nascita del romanticismo tedesco, poichè in quell'anno vide la luce la rivista Athenaeum, fondata da $\mathrm{Fe}$ derico Schlegel, il più originale fra i teorici della poetica romantica. Augusto Guglielmo Schlegel, fratello del precedente, vi collaborò appassionatamente, svolgendo di preferenza gli aspetti storici e letterari della scuola, rendendosi celebre col suo Corso di letteratura drammatica, che fu tradotto rapidamente nelle principali lingue europee.

Novalis è il più grande poeta romantico del primo periodo. La sua poesia è sognante, nostalgica, visionaria. Diede alla Germania quei nuovi mondi poetici di cui andava in cerca, e vide nel "fiore azzurro" il simbolo dell'ideale romantico. "A flor azul é o símbolo da poesia romântica. Azul é a côr da longitude infinita e, assim, a expressão da saudade. Este sentimento de saudade é o sentimento básico do romantismo" (21). Poco per volta, ma rapidamente, tutti entrarono nell'orbita del movimento, che dilagò ovunque in marcia trionfante. Tieck cantò il suo amore per la natura selvaggia, per le foreste, per i paesaggi lugubri e impressionanti; la poesia sensuale e mistica di Brentano celebrò le stelle ed i fiori, l'amore e la sofferenza, le saghe antiche e le favole popolari; Eichendorff diede vita al fascino delle selve germaniche, inneggiò ai viaggi fantastici, alle acque ed ai venti; Goerres ruggi come un leone in difesa delle libertà politiche

20) Giovanni ECKERMANN, Colloqui col Goethe (tr. it.), Bari, 1912-1914, vol. II, p. 28.

21) Mansueto KOHNEN, História da Literatura Germânica, Impressora Paranaense, Curitiba, 1949, vol. II, p. 16. 
e religiose; Uhland con le sue ballate fece rivivere nella fantasia dei connazionali l'intensa vita della movimentata Germania medievale.

Un altro aspetto, fortemente accentuato, del romanticismo tedesco è il sentimento della grandezza germanica e della necessità di liberare la patria dallo straniero. "A germanidade, que venceu sob Hermann, o Cherusco, as legiões romanas e que realizou na Idade Média a grande idéia do Reich, reluziu qual estrêla luminosa e Leitmotif para o futuro Estado nacional" (22).

La Germania, come il resto d'Europa, era allora dominata dalla volontà dispotica di Napoleone, che, nel campo dell'arte, favori il considdetto neoclassicismo. Sicchè il movimento romantico tedesco ingaggiò simultaneamente la lotta contro il neoclassicismo e l'oppressione straniera. Divenne cosi il portavoce dell'anelito tedesco per la libertà. I poeti romantici furono tutti patrioti e le loro canzoni echeggiavano in ogni canto della Germania come uno squillo di redenzione e di libertà: libertà dai legami politici stranieri, e libertà dagli impacci artistici che impedivano alla nazione di avere una grande letteratura di contenuto autoctono.

Le principali figure che svilupparono questo aspetto nazionale del romanticismo furono: Fouqué, autore di canzoni militari; il già ricordato Eichendorff, poeta e soldato allo stesso tempo; Arndt, poeta della libertà tanto in patria che in esilio, confortando il popolo sofferente con la suprema formula della religiosità cristiana; Koerner, il poeta eroe caduto sul campo di battaglia combattendo contra il nemico della Germania; Kleist, bardo profetico della liberazione nazionale, sebbene morto prima di poterla vedere. Simili squilli di tromba guerriera e accenti di sospirata libertà risuonarono pure nella multiforme poesia di Schenkendorf, Rueckert, Arnim, ed altri ancora.

Anche in Inghilterra il romanticismo ha una data con-

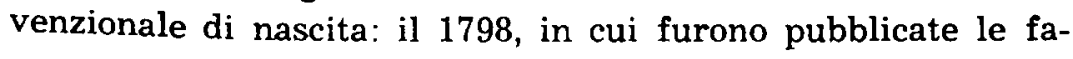


mose Lyrical Ballads di Wordsworth e Coleridge. Ma anche là questo movimento era stato preparato da lunga data, specialmente con la pubblicazione dei poemi del ciclo di Ossian, l'eroe leggendario e bardo gaelico, che si suppose vissuto nel terzo secolo dell'era cristiana. Questi canti popolari furono tradotti assai liberamente da Macpherson, inserendovi passi di sua invenzione; pubblicati ripetutamente, suscitarono dentro e fuori d'Inghilterra un entusiasmo straordinario. Anche i poeti inglesi trovavano cosi una epopea a sfondo autoctono, che, poco per volta, orientò la letteratura verso la celebrazione delle antiche grandezze patrie, $e$ in seguito diede origine al movimento romantico inglese, sotto la profonda influenza dei romantici tedeschi.

I canti ossianici, molto uniformi, "narrano una grande quantità di storie assai intricate, i cui motivi dominanti sono la guerra, l'incredibile virtù cavalleresca dei guerrieri, il melanconico destino di varie coppie di amanti o di sposi, con episodi di ratto, di caccia, di tempesta, e soprattutto descrizioni, assai fresche, di paesaggi, con verdi e cupe distese di mare, laghi e brughiere solitarie... I poemi spirano una profonda malinconia, un sentimento di vanità di sogni e di speranze, di tragica fine d'ogni amore, e insieme l'emozione suscitata dalle reliquie del passato. Così questa poesia s'armonizzava perfettamente col gusto romantico che s'andava maturando in quegli anni" (23).

Robert Burns e William Blake furono i due primi grandi poeti del romanticismo inglese. Il primo ebbe una vita molto sregolata. Nella sua arte entrano i soliti temi romantici e scatti di rivoluzionario antitradizionalista inneggiante alla libertà del popolo. Il secondo, singolarissimo temperamento di poeta visionario - affermava d'aver colloqui corporali con i santi e con i profeti - alzò la bandiera della "sacra insurrezione", proclamando di non conoscere altro vangelo "che

23) Mario PRAZ, Storia della Letteratura Inglese, Sansoni, Firenze, 1944. p. 242 . 
la libertà corporale e spirituale di esercitare le divine arti dell'Immaginazione".

Ma furono le Lyrical Ballads di Wordsworth e Coleridge che diedero il via al romanticismo inglese, segnando un "vero e proprio rinascimento del senso di stupore elementare di fronte al mistero del mondo"' (24). Nelle isole britanniche il romanticismo non ebbe accentuate effervescenze patriottiche come in Germania; i primi entusiasmi suscitati dalla Rivoluzione Francese lentamente svanirono davanti alle mire imperialistiche della Francia napoleonica, che fecero retrocedere $i$ romantici inglesi verso posizioni più conservatrici.

Scomparso il pericolo dell'egemonia napoleonica, i fermenti romantici che volteggiavano sulla letteratura britannica si orientarono piuttosto contro le forme viete e retrive della vita aristocratica. $\mathrm{Ma}$ lo spirito eccessivamente immorale ed anarchico dei due più famosi poeti romantici inglesi - Byron e Shelley - attirò loro l'ostracismo in patria. I cantori di Aroldo e di Prometeo esularono verso il continente, attratti specialmente dal fascino malioso di Roma ed Atene. Due dei grandi romantici inglesi morirono in Italia, Shelley e Keats. Quest'ultimo, ammaliato dalla visione romantica dell'antica arte classica, intraprese un viaggio sentimentale verso l'Italia e la Grecia per poter contemplare da vicino quei monumenti antichi che esercitarono sulla sua fantasia una forza d'incantesimo.

I due romantici inglesi che maggior influenza ebbero sugl' italiani furono Byron e Scott, sebbene i critici italiani stentino a vedere dei vantaggi veramente benefici sulla letteratura italiana da parte dei due romantici mentovati (25). "Mentre l'influsso di Byron in Inghilterra fu vasto solo sui minori e sui minimi, e nullo, o debole, o presto superato su

24) Mario PRAZ, Storia della Letteratura Inglese, cit., p. 264.

25) Scrive, infatti, Mario PRAZ nei Rapporti tra la letteratura italiana e la letteratura inglese: “... a parte il romanzo storico del Manzoni, quale altra opera memorabile troviamo nella pullulante schiera d'imitatori e raffazzonatori dei due celebri inglesi?" Cf. Letterature Comparate, Marzorati, Milano, 1948, p. 185. 
quelli che dovevano essere $i$ veri poeti futuri, in italia, come in genere sul Continente, il Byron sali subito a una fama superiore a quella di qualsiasi altro scrittore straniero... I romantici italiani inneggiavano al Byron come a maestro... Il Monti affermava essere il suo romanticismo d'un genere così sublime che Omero stesso gli avrebbe perdonato" (26).

Si deve tuttavia notare che questo influsso non durò a lungo, e già i più fini intenditori d'arte dell'epoca, come, per esempio, Leopardi e Tommaseo, mostrarono di non lasciarsi ingannare dalla "vistosità di quella Musa" (27).

Anche in Francia il romanticismo nacque prima che in Italia. Come anno di nascita è preso il 1813. In quell'anno, con la battaglia di Lipsia. non solamente cominciò il declino del potere di Napoleone, che aveva favorito il neoclassicismo, ma vide anche la luce il famoso libro di Madama Staël De l'Allemagne, e fu tradotto il Corso di letteratura drammatica di A. G. Schlegel. La Staël era avversaria del despotismo napoleonico, e quindi, anche nel campo nell'arte, invitava i suoi connazionali a liberarsi dalle viete regole neoclassiche, fino allora dominanti in Francia. I letterati francesi avrebbero dovuto imitare quelli tedeschi, che erano già ben avanti sul cammino della nuova arte romantica, e davano all'Europa l'esempio di una letteratura fresca, indipendente e vigorosa. In verità, le idee di Rousseau avevano, in certo senso, già preparato gli spiriti per l'inizio della letteratura romantica, col predicare la belezza e la forza della natura istintiva a cui l'umanità doveva ispirarsi. Sicchè, al tramonto repentino della stella napoleonica, il romanticismo divenne la forma letteraria dominante. La possente voce di Chateaubriand esercitò un fascino straordinario, tanto più che la sua vena romantica si ergeva contra l'assolutismo napoleonico e si schierava in difesa del cattolicismo.

26) Mario PRAZ, in Problemi ed orientamenti critici di lingua e letteratura italiana - Letterature Comparate, Marzorati, Milano, 1948 p. 185. 
Il romanticismo francese, più tardivo di quello tedesco e inglese, sebbene ispirato anch'esso ai principi della poetica romantica, presenta caratteristiche fortemente indiduali nei vari letterati e poeti. Specialmente la lirica, il romanzo, la storia, il teatro furono i generi cari ai romantici. Così, esponenti di quell'alta lirica che si espande nel canto, furono Lamartine, Hugo, Musset, Vigny; passione per il romanzo dimostrarono, oltre Chateaubriand, Sénancour, Constant, Hugo, Vigny, Sand, Sainte-Beuve, Stendhal; amore per la ricerca storica manifestarono Guizot, Thierry, Michelet.

I due romantici francesi più conosciuti in Italia sono Lamartine e Hugo. Il primo ebbe rapporti molteplici con la penisola, dove sostenne perfino un duello con Gabriele Pepe, causato da poco rispettose parole verso gl'italiani; il secondo fu specialmente ammirato per le sue idee politiche e sociali.

Il romanticismo italiano, storicamente, è posteriore a quello germanico, inglese e francese. Quando spuntò sull'Italia l'astro romantico, esso splendeva già da tempo sui cieli d'Europa (28).

\section{4. - Nascita e trionfo del romanticismo in Italia.}

Il romanticismo italiano deve cercare le sue origini in Germania, in Inghilterra ed in Francia. Tuttavia in Italia questo movimento letterario e culturale non poteva disconoscere il passato, a cui gl'italiani non potevano abdicare senza andare incontro ad una specie di suicidio. Se per i tedeschi e gl'inglesi abbandonare il mondo classico per ricercare ispirazione poetica nella loro storia dell'alto Medio Evo, o dei tempi ancor più remoti, poteva significare uno sforzo per potenziare il sentimento nazionale, ciò non aveva senso per gl'italiani, la cui storia era intimamente legata all'antichità greco-romana. Non potevano, quindi, gl'italiani rinnegare completamente l'arte classica. Patrocinarono, tuttavia, un rinnovamento che permettesse anche a loro di inserirsi nella grande corrente romantica che aveva invaso l'Europa. "Se-

28) Cf. Letterature Comparate, elt., in relazione alla letteratura inglese, tedesca, francese, spagnola. 
guiteci - dicevano i romantici d'ogni paese - per i sentieri fioriti di sogni e d'incanti che dal presente risalgono verso il passato; vi mostreremo le origini remote e le ragioni nobilissime delle idee che la schiera perversa dei filosofi e degli enciclopedisti settecenteschi ha giudicato irrazionali" (29). Quest'invito fu ascoltato dagli italiani, che si affiancarono, così, ai romantici d'oltr'Alpe.

Il romanticismo italiano ebbe, più ancora del tedesco, profondo carattere politico e nazionale. Esso si credette investito della grande e nobile missione di dover portare l'Italia alla liberazione dallo straniero e all'unione nazionale.

Gli storici della letteratura italiana prendono il 1816 come anno di nascita del romanticismo peninsulare, poichè in quell'anno accaddero alcuni fatti storicamente e letterariamente famosi, decisivi per il trionfo dell'idea romantica in Italia. Li ricorderemo più sotto. Tuttavia, anche in Italia, si vollero vedere dei precedenti del romanticismo in scrittori di epoche antiche. Il languoroso sentimento amoroso del Petrarca parve a più d'uno un pretesto per giustificare l'origine autoctona, domestica, del movimento romantico. Certo è, però, che gli scrittori del Caffè, per la loro passione rinnovatrice e per la loro ribellione alle raggelate forme artistiche di una poética retriva e pedante, si possono considerare gl' immediati preparatori del romanticismo peninsulare. Infatti, le pagine del famoso giornale lombardo agitavano due idee fondamentali che saranno riprese dai romantici come principi programmatici: propositi di modernità a tutti i costi nel campo letterario, aderendo più strettamente ad uma forma di poesia popolare, e l'anelito di unire tutti gl'italiani in una sola patria comune, unita e indipendente. Dal Baretti discese ai romantici la ventata libertaria e antiarcadica, e dall'Alfieri lo spirito vigoroso di lotta contra i tiranni, cioè, contro gli oppressori d'Italia.

Nel 1816 apparve sul primo numero del periodico mila-

29) Alfredo GALLETTI, Natura e finalita della storia nel moderno pensiero europeo, Fabbri, Milano, 1953, p. 39. 
nese La Biblioteca italiana il discorso di Madama Staël: Sulla maniera e la utilità delle traduzioni. Strano davvero che sia stata una scrittrice francese a fare il primo invito, diciamo cosi, ufficiale e pubblico agl'italiani affinchè si risolvessero a studiare e ad imitare i romantici oltremontani; più strano ancora che il celebre discorso sia stato tradotto da Pietro Giordani, classico ad oltranza, e sia stato pubblicato su di una rivista che doveva essere il portavoce del classicismo e del sentimento politico austriacante. La Staël invitava gl'italiani a rinnovare lo spirito della letteratura italiana, attingendo nuova linfa alle sorgenti della fresca poesia romantica straniera, particolarmente tedesca. Ma non tutti accettarono con benevolenza l'invito. Vincenzo Monti fece sentire la sua voce in difesa del classicismo, e Giacomo Leopardi, allora quasi ignoto, inviava una lettera $\mathbf{A i}$ compilatori della Biblioteca italiana, i quali non la pubblicarono. Apparve postuma fra gli scritti leopardiani e così sappiamo che essa riconosceva l'utilità della conoscenza degli scrittori stranieri per gli scienziati, ma non per i letterati, "perchè le scienze possono fare e fanno progressi tutto giorno, dove la letteratura non può farne". L'imitazione degli stranieri poteva essere nociva, poichè nelle loro opere si trovano "esagerazioni ed immaginazioni gigantesche, ed assai raramente la vera castissima santissima natura". E, dopo altre varie osservazioni, così concludeva l'allora sconosciuto contino di Recanati: "Io come Talete ringraziava il Cielo per averlo fatto Greco, ringraziolo di cuore per avermi fatto Italiano, nè vorrei dar la mia patria per un Regno, e ciò non per il potere d'Italia, che niuno ne ha, nè per il suo bel clima di cui poco mi cale, nè per le sue belle città di cui mi cale ancor meno, ma per lo ingegno degl'Italiani, e per la maniera della italiana letteratura che è di tutte le letterature del mondo la più affine alla greca e latina, cioè a dire (parlo secondo la mia opinione ed altri segua pure la sua) alla sola vera, perchè la sola naturale, e in tutto vota d'affettazione". 
Incominciò cosi la polemica classico-romantica, che doveva protrarsi per molti anni e terminare col trionfo della scuola romantica italiana.

Alle idee romantiche aderì con pronta decisione Giovanni Berchet (30), e nel 1816 pubblicava la famosa Lettera semiseria di Grisostomo, che diede definitivamente il via al movimento romantico italiano. La Lettera, nonostante i suoi limiti e le sue deficienze, racchiude un nucleo di verità sane e nuove, che, a quell'epoca. fecero grande impressione. La nuova poesia italiana deve essere popolare, come quella tedesca, e non deve ispirarsi alla mitologia antica, che non dice più nulla ai moderni; quelli che vogliono cantare $i$ costumi, le passioni, le opinioni degli antichi, fanno una poesia morta; quelli che, al contrario, celebrano la natura, la credenza del popolo, i sentimenti del cuore, le aspirazioni moderne, fanno una poesia viva. "La poesia de' primi è classica, quella dei secondi è romantica... io stimo di poter nominare con tutta ragione poesia de' morti la prima, e poesia de' vivi la seconda. Nè temo d'ingannarmi dicendo che Omero, Pindaro, Sofocle, Euripide ecc. ecc., furono al tempo loro in certo modo romantici, perchè non cantarono le cose degli Egizi o de' Caldei, ma quelle dei loro Greci; siccome il Milton non cantò le superstizioni omeriche, ma le tradizioni cristiane... l'anima è commossa al vivo dalle cose nostre che ci circondano tutto dì, non dalle atiche altrui, che a noi sono notificate per mezzo soltanto de' libri e della storia".

Dire che l'Italia non può avere una letteratura nazionale perchè non è unita politicamente, è un grave errore. Se l'Italia non esiste come nazione unita, chi potrà impedire che essa si crei, fin d'ora, una letteratura nazionale? Forse che Dante, Petrarca, Ariosto e tutti gli altri grandi scrittori italiani dei

30) Giovanni BERCHET (1783-1851) fu scittore poeta, patriota e deputato al Parlamento di Torino. Andò in isilio in Svizzera e a Londra. Oltre la sua attività come teorico dei principi romantici, si distinse per numerose traduzioni, tra cui famose sono quelle dei due componimenti poetici del Bürger Il cacciatore feroce ed Eleonora. 
secoli passati aspettarono che l'Italia fosse unita per risolversi a scrivere in italiano?

Berchet, quindi, butta via tutte le altre poetiche come vecchi impacci, ed esorta tutti gl' italiani a formare una letteratura civile, nazionale, moderna, cristiana. $\mathrm{Si}$ comprende che una voce cosi radicalmente innovatrice doveva destare immenso scalpore nelle schiere letterarie. Non mancarono coloro che vollero metter pace fra i litiganti, tentando conciliare classici e romantici, con l'esito di non contentare nessuno ed acuire ancor più la contesa. Tra gli altri, Giuseppe Londonio tentò pacificare i campi oposti con i Cenni critici sulla Poesia romantica, usciti nel 1817. Ma non contentò nessuno, come accade a quelli che tentano mettere pace fra cose inconciliabili. Al Londonio rispose un giovane abate torinese pieno di talento, Ludovico di Breme (31), uno dei primissimi romantici, circondato da uno straordinario prestigio. "Primo frutto di questa scienza nuova - replicava egli al Londonio - sarà di abolire per sempre le grette convenzioni sopra di cui riposa l'attuale pedagogia letteraria e le attuali discipline poetiche, più Galliche per certo che Italiche, e meno Greche d'assai ch'altri non s'avvede. Impareremo dai Greci, e da quanti furon grandi nei secoli di poi, a non ricopiare mai più nè Greci, nè Latini ma bensì ad emularli, gareggiando secondo i vari tempi, e nello spaziare generosamente e grandiosamente per l'immensità del cuore umano" (32).

Il movimento letterario romantico italiano ebbe le sue origini nel nord della penisola; i centri più importanti furono Milano, Torino e, dopo, anche Firenze. Per la prima volta nella storia delle lettere italiane, l'Italia settentrionale passò all'avanguardia. Il Trecento ebbe il suo centro ideale in Firenze, e toscani furono i tre grandi trecentisti Dante, Petrarca, Boccaccio. Il Rinascimento ebbe i suoi centri d'irra-

31) Ludovico DI BREME $(1780-1820)$, illustre figura del patriziato piemontese, fu uno dei primissimi fautori del romanticismo; si dedicò piuttosto alla critica e alla diffusione delle idee romantiche, anzi che alla produzione strettamente letteraria.

32) Ludovico DI BREME, Polemiche, UTET, Torino, 1928, p. 112. 
diazione in Roma, Napoli e Firenze. L'Ottocento romantico cominciò in alta Italia e di là si estese quasi subito a Firenze e quindi altrove. Questo è un fatto nuovo, senza dubbio, e deve essere attribuito allo spirito nazionalista che nel Piemonte ed in Lombardia fu più intenso che altrove; gli scrittori romantici, oltre gl' ideali strettamente letterari e ideologici, aveano pure quello patriottico e nazionale. Tanto politicamente che letterariamente. fu l'Italia settentrionale che si mosse per prima nella grande campagna risorgimentale che portò all'unificazione e all'indipendenza italiana.

A Milano, nel 1818, vide la luce il Conciliatore, il foglio azzurro che, in letteratura. fu il portavoce del romanticismo e, in politica, l'espressione del liberalismo, più o meno cattolico, desideroso di unificare l'Italia. Fu una campagna vivace e gagliarda quella del Conciliatore, fondato dal Conte Luigi Porro. Vi collaborarono con entusiasmo e dedicazione i già citati Di Breme e Berchet, il Pellico, il Borsieri, il Confalonieri, il Romagnosi, il Visconti e molti altri (33). Oltre la letteratura, anche la politica, le scienze sociali, la filosofia trovarono ospitalità nelle sue pagine. Osteggiato dal governo austriaco (34), cessò di uscire dopo tredici mesi di vita. Ma le idee che diffuse portarono dovunque un fermento che doveva dare i suoi frutti. Non pochi degli scrittori del giornale finirono nelle prigioni austriache, martiri dell'indipendenza italiana. Di questi il più illustre fu Silvio Pellico (35), il quale scrisse essere divenuta allora opinione comune e corrente che romantico significasse liberale e patriotta, "nè più osarono dirsi classicisti fuorchè gli ultra e le spie".

33. E interessante notare che il primo numero del Conciliatore pubblicava un elogioso articolo sul poema di Camōes, Os Lusiadas, occasionato dall'edizione che ne curó, in Parigi, J. M. de Scuza Botelho, nel 1817.

34) Il Governo austriaco di Milano creò un nuovo giornale L'accattabrighe, che fu chiamato "foglio roseo" dal colore della carta e fece opposizione al Conciliatore, chiamato "foglio azzurro". L'accattabrighe era diretto dal conte Caleppio, commissario di polizia.

35) Silvio PELLICO $(1789-1854)$ fu una delle più nobili fugure del romanticismo e del Risorgimento italiano. Ancora oggi quasi tutti gli italiani leggono Le mie prigioni, dove il Pellico racconta la storia della sua prigionia nella fortezza dello Spielberg. 
Al romanticismo diedero pure un grande contributo $i$ due più insigni poeti dialettali del secolo: Carlo Porta (36) a Milano, e, più tardi Gioacchino Belli (37) a Roma. Il primo scrisse con ricca vena in dialetto milanese, ed il secondo, con inesauribile fecondità di sonettista, in dialetto romanesco.

Poco per volta quasi tutti gli scrittori entrarono nelle file romantiche: Manzoni, Cantù, Grossi, Torti, Rosmini, Tommaseo, Carrer. A Torino si formò un altro centro romantico, con tendenze piuttosto storiche e politiche, i cui nomi più famosi sono quelli del Santarosa, D'Azeglio, Balbo; a Firenze si schierava in favore del romanticismo l'Antologia del Vieusseux, e romantici furono i famosi giornali L'Indicatore Livornese e L'Indicatore Genovese.

Dopo i tristemente celebri processi del 1821, che condannarono molti romantici italiani per le loro idee di libertà politica, la marcia del romanticismo peninsulare ebbe una stasi momentanea ed i classicisti parvero alzare la testa quando il Monti pubblicò, nel 1825, il Sermone sulla mitologia, e la scuola classicheggiante romagnola diede qualche guizzo di vita col suoGiornale Arcadico, le cui pubblicazioni erano state iniziate nel 1819. Ma nel 1827 uscirono I Promessi Sposi del Manzoni, i cui Inni Sacri erano già popolarissimi; nello stesso anno moriva a Londra, abbandonato da tutti e vittima precoce della sua vita sregolata, Ugo Foscolo, ritenuto un classicista; nel 1828 moriva l'altro vate del classicismo, Vincenzo

36) Carlo PORTA $(1776-1776)$, il più grande poeta dialettale del suo secolo, dopo aver studiato con i Gesuiti a Monza, entrò in seminario, ma ne usci in seguito e divenne un anticlericale. Si schierò fra i romantici, scrivendo le briose sestine El romanticismo.

37) Giuseppe Gioacchino BELLI (1791-1863), fu il più grande poeta romanesco del suo secolo. "impiegato del governo pontificio, che per tutta la vita professò nòn soltanto la più ortodossa fede cattolica, ma anche le idee ufficiali". Cf. A. Pompeati, op. cit., p. 243. Sembrò vergognarsi dei suoi sonetti romaneschi per lo spirito anticlericale che in essi aleggiava. Li affidò a Monsignor Tizzani perchè li bruciasse. Furono invece pubblicati postumi. Belli non fu un romantico militante; tuttavia il suo mondo si aggira nella sfera romantica e il Flora lo collocò tra i ploeti dell'età romantica perchè la sua poesia "si alimentò sopra tutto di spiriti romantici". Cf. F. FLORA, Storia della letteratura italiana, Mondadori, Milano, 1953, vol. IV, p. 333. 
Monti; la poesia del Leopardi, morto a Napoli nel 1837, se era considerata classica per la sua purezza formale veramente perfetta, presentava una "estrema posizione romantica" (38) nella maggior parte dei temi che ricorrevano nelle sue canzoni.

Il Manzoni portò al romanticismo un contributo, che fu decisivo, col prestigio grandissimo della sua persona e con la limpidezza della sua arte. Aveva già manifestato la sua avversione ai classicisti, nel 1816, con uno scherzo poetico intitolato L'ira d'Apollo, e, più tardi, formulò i suoi principi romantici nella Lettera sul romanticismo (39). Dove, però, riusci ad inverare in opere artistiche la sua vocazione romantica fu negli Inni Sacri e nei Promessi Sposi. Questo romanzo è il capolavoro della prosa italiana, "uno dei libri più multiformi della letteratura universale", dove non è possibile incontrare neppure un "motivo fallito" (40", e che Francesco De Sanctis, il maggior critico fiorito alla scuola del romanticismo, defini con una formola famosa: "il limite dell'ideale". Il Manzoni non entrò nel gruppo del Conciliatore, sulle cui pagine non apparve mai alcun articolo del celebre poeta e prosatore milanese. "Difensore pacato, ma tenace della propria libertà - scrive Arturo Pompeati -, il Manzoni doveva essere uno di quei signori di razza, compitissimi e sorridenti, che pure riescono con un nulla a segnare una linea invalicabile fra sè e i vari esemplari dell'indiscrezione umana... Non avvicinò il Byron, lo Stendhal, la Staël, lo Schlegel, durante i loro soggiorni milanesi" (41). Tuttavia militò nelle file romantiche, e ne divenne il maggior campione, sebbene non condividesse tutti i punti di vista di quella tumultuante scuola letteraria. E che il Manzoni era un genio autentico e nei suoi capolavori non poterono entrare le deficienze dell'arte romantica.

38) Walter BINNT, La nuova poetica leopardiana, Sansoni, Firenze, 1947, p. 88.

39) Fu scritta nel 1823 e diretta al marchese Cesare Taparelli D'Azeglio. Non era destinata al pubblico, ma, dopo la morte del D'Azeglio, fu data alle stampe, nel 1846.

40) Attilio MOMIGLiaNO, Storia della letteratura italiana, cit., p. 467.

41) Arturo POMPEATI, op. cit., p. 103. 
Quasi tutti i generi letterari furono trattati dai romantici, naturalmente con abilità e sensibilità artistica diversa; qualche volta perfino con esito negativo. Oltre il Manzoni, scrissero romanzi storici il Grossi, il D'Azeglio, il Cantù, il Guerrazzi, il Nievo, il Bresciani, il Rovani; la novella in versi ebbe i suoi rappresentanti nel Grossi, Cantù, Sestini, Pellico, Torti; la poesia satirica, la lirica, la romanza, la ballata, tutte forme poetiche predilette dai romantici, furono coltivate rispettivamente dal Porta, Belli, Brofferio, Fusinato, Giusti, Manzoni, Berchet, Carrer, Grossi, Sestini, Cantù, Tommaseo, Poerio, Mameli, Mercantini, Parzanese; il teatro tragico fu rappresentato dal Manzoni, dal Pellico; la letteratura autobiografica ricorda i nomi del D'Azeglio, Pellico, Giusti, Settembrini; la storia, la politica, la filosofia, la critica ci presenta i nomi del Balbo, Manzoni, Cantù, Settembrini, Tommaseo, Rosmini, Gioberti, Mazzini. Nè si deve dimenticare che il più grande critico letterario dell'Ottocento romantico è Francesco De Sanctis, fiorito quando il romanticismo era già verso la sua fine.

Questa folta schiera di nomi ci fa comprendere la portata, l'interesse, l'intusiasmo che destò in Italia il movimento romantico e l'influenza che esso esercitò sulla cultura e sulla vita. Col Pellico rinnovò il fervore religioso e difese il valore della persona umana; col Manzoni mise in luce l'intervento della Provvidenza divina nelle vicende umane e conciliò alla patria l'animo dei credenti; col Rosmini esaltò la forza operosa della volontà e celebrò la dignità etica e religiosa della vita; col Gioberti ingaggiò la lotta contra il despotismo per la difesa delle libertà nazionali; col Mazzini fece udire il verbo patriottico in difesa dei popoli oppressi.

In seguito, quando al movimento romantico vennero a mancare forti ingegni e nobili caratteri, esso diede passi falsi e degenerò apertamente verso zone di sentimento oscuro e torbido, esaurendo quelle forze sane che gli avevano dato il primigenio impulso costruttivo. "Spesso il sentimento degenerò in languido sentimentalismo, e la sovraeccitazione della 
fantasia creò false rievocazioni di vita medievale; scene fosche e paurose e lugubri visioni di tregende infernali, di spettri e di streghe, nelle quali al tragico si sostituisce l'orrido e il grottesco, e alla fede la superstizione, e le immagini larvate di misticismo mal celano talvolta una morbosa sensualità. Ma queste aberrazioni del romanticismo, per lo più di derivazione nordica, contro le quali già protestava il Berchet, non durarono a lungo in Italia" (42).

Queste caratteristiche negative del romanticismo italiano fanno irruzione selvaggia specialmente nei due periodi che gli storici della letteratura italiana chiamano col nome di secondo e terzo romanticismo.

\section{4. - Secondo e terzo romanticismo italiano.}

I difetti che erano latenti nella dottrina romantica diedero subito i loro frutti nella prolissa produzione letteraria di due poeti che, per unanime opinione dei critici, sono gli esponenti del cosiddetto secondo romanticismo: Giovanni Prati e Aleardo Aleardi.

Il Prati volle essere il poeta del cuore e del sentimento; ma il suo fu un pessimo atteggiamento. Il poeta, secondo lui, è un uomo diverso dagli altri mortali, un essere "privilegiato da una sorte irrevocabile, triste $e$ inebriante, affascinante $e$ fatale, passa fra i mortali con l'occhio rapito, il gesto ieratico, la parola ispirata, profeta dei destini umani" (43). Anche l'aspetto fisico con cui si presentavano questi bardi romantici tardivi - che ai loro tempi sembrarono geni brillanti, ma ritenuti ora in qualità di ridicoli e goffi - era per lo più "alquanto teatrale, con fieri pizzi e mustacchi e una gran chioma ondante e lucente" (44).

Il Prati scrisse con facilità torrenziale e di ogni cosa fece poesia, conquistando una fama clamorosa sebbene effimera. Nella sua poesia s'incontra una ridda vertiginosa di

42) Domenico DE PAOLI, in Grande Dixionario Enciclopedico, UTET, Torino, 1938, vol. IX voce Romanticismo.

43) A. POMPEATI, op. cit.. p. 316.

44) Idem, ibidem, p. 317. 
castelli, dame, cavalieri, amanti, fughe, notti lunari, foreste, galoppate, tornei, uccisioni; l'uniformità e l'intrinseca povertà dell'animo la fecero cadere presto di moda, a ciò contribuendo anche la critica, che la defini giustamente una poesia "impura, pletorica e torbida".

L'Aleardi fu poco diverso dal Prati, sebbene più abile ed aristocratico nel cesellare il verso, specialmente quello sciolto, che seppe maneggiare con destrezza e bravura. Cantò la pa- . tria e la donna, conquistando un grande numero di lettori e più ancora di lettrici. Sebbene non si possa negare a questi due verbosi poeti qualche felice momento di ispirazione, nel complesso furono artisti mancati; troppi voli fecero per il cielo della poesia, ma nessun volo li portò tanto in alto da collocarli al fianco dei grandi poeti italiani, nè riuscirono a darci un capolavoro. Bene sentenziò di loro Francesco Flora, quando scrisse che l'arte del primo (Prati) è una "pronta eccitazione canora" a cui manca "una vera forma di canto", nonostante che affiori qua e là "qualche immagine ispirata, qualche melodia romita" (45), e vide nella prodiga cordialità verbale del secondo (Aleardi) "sentimenti e immagini" privi di "coscienza artistica", sfoghi estemporanei "ai quali manca l'intensità e la durata" (46).

Il terzo romanticismo italiano è conosciuto anche col nome di "Scapigliatura". Questo movimento tardivo del romanticismo peninsulare irruppe scompostamente tra il 1860 e il 1870 , qual ribellione contro la moda romantica in voga, che era quella del Prati e dell'Aleardi. L'astro poetico carducciano era già alto sui cieli d'Italia, e faceva sentire altre esigenze letterarie.

Inventore del nome fu Carlo Righetti. "Scapigliato" doveva significare, secondo la mentalità della scuola, "boemo", cioè colui che conduce una vita libera e disordinata. I rappresentanti della "scapigliatura" milanese furono: Rovani, 
Righetti, Praga, Camerana, Tarchetti, Pinchetti e Dossi. Tentarono ispirarsi ad alcuni letterati d'oltr'Alpe, specialmente a Baudelaire, Hugo e Heine; dal primo pretesero imitare il satanismo sbrigliato e sensuale, dal secondo la sonorità della frase sentenziosa e tribunizia, dal terzo l'ironia e la beffarda insolenza. Mancava loro, però, il genio dei tre grandi oltramontani. Furono caretteristiche degli "scapigliati": versatilità artistica, perciò si applicarono a più arti simultaneamente; sentimento desolato della vita dell'uomo sempre oscillante tra la virtù ed il vizio, tra il bene e il male, tra Dio e Satana. Nessuno di loro riuscì ad essere vero grande poeta, e sebbene ostentassero certa opposizione al romanticismo, in realtà finirono per concretizzare nella loro produzione letteraria le più forsennate ed estreme tendenze libertarie della scuola romantica.

Scapigliati, cioè, disordinati furono realmente, tanto nella vita che nella letteratura, tanto che, quasi tutti, morirono suicidi o consumati precocemente dai vizi. Rovani si degradò nel vizio dell'assenzio e morì in una casa di salute; Righetti fu bruciato dall'uso dell'assenzio; Praga morì consumato dall'alcool; Tarchetti fu portato via dalla tisi dopo una vita allucinata; Camerana, che $\mathrm{fu}$ anche magistrato, si uccise; Pinchetti fini suicida, come pure altri poeti minori del gruppo; Dossi mori abbandonato e solitario. Come si vede, l'ipilogo degli "scapigliati" fu davvero tragico e disperato. Arrigo Boito, che fece parte, per qualche tempo, della "scapigliatura", tracciava lo spirito e l'ideale di questi libertini ribelli e disperati con $i$ versi seguenti:

Torva è la Musa. Per l'Italia nostra corre levando impetuosi gridi

una pallida giostra

di poeti suicidi.

Alzan le pugna e mostrano a trofeo dell'Arte loro un verme ed un aborto,

e giocano al paleo

colle teste da morto. 
Io pur fra i primi di cotesta razza

urlo il canto anatemico e macabro,

poi, con rivolta pazza,

atteggio a fischi il labro.

Il movimento romantico italiano, dopo una lunga e gloriosa storia, ricco già d'uomini illustri, si esauriva in questo misero modo.

5. - Aspetti negativi e positivi del romanticismo italiano.

L'Ottocento romantico presenta alcuni aspetti negativi, dovuti, in parte, ai limiti della dottrina poetica romantica e, in parte, alle deficienze artistiche, temperamentali ed etiche di non pochi suoi fautori.

Tra gli aspetti negativi si debbono certo annoverare: le intemperanze della fantasia, lo spirito di ribellione e di libertà portato fino al parossismo, l'individualismo esasperato e anarchico, la predilezione per gl'incantesimi e le fiabe del Medio Evo, il sensualismo brutale che svincolò l'amore dalle sane leggi dell'etica, il falso misticismo che indusse non pochi romantici a considerare il dubbio "più poetico dellá certezza" e vedere nel mistero un mezzo per fare "più stupendo lo spettacolo dell'universo" (47).

Accanto, però, a questi aspetti negativi, il romanticismo italiano ne ha pure altri positivi. La maggior parte dei romantici italiani furono sinceri patriotti e spiriti profondamente religiosi, "ben fermi in certi principi di rigida moralità, in una costante coerenza di sentimento e di pensiero, di idealismo artistico e di volontà pratica che conferì alle loro opere una efficacia veramente educatrice" (48).

Il romanticismo rinnovò la letteratura italiana, liberandola dai secolari languori dell'Arcadia, dall'anacronismo della mitologia; fece conoscere meglio in Italia le letterature straniere e promosse una poesia popolare più compresa dai lettori,

47) Alfredo GALLETTI, Natura e finalità della storia nel moderno penslero europeo, cit., p. 71.

48) Alfredo GALLETTI, Storia letterarla d'Italia, vol. X, I Novecento, F. Vallardl, Milano, p. 31. 
aderente alle necessità vive e moderne; favorì lo studio della storia; mise in luce la bellezza poetica del mondo cristiano; permise una più ampia libertà nella struttura della tragedia e nei mezzi di espressione; svolse una parte preponderante nel movimento nazionale che portò l'Italia all'unità politica; creò forme nuove di poesia ed una prosa artistica più limpida, snella e moderna. Sicchè l'Ottocento romantico si deve considerare, dopo il Trecento, il periodo più ricco e fecondo della letteratura italiana. I Promessi Sposi di Alessandro Manzoni sono il più bel fiore del romanticismo italiano e la profonda influenza artistica ed educatrice che questo romanzo esercitò su tutte le generazioni posteriori è tale che - al di là dell'Ariosto e del Boccaccio - richiama alla Divina Commedia di Dante Alighieri.

\section{RETIFICACAO \\ Uma Cronologia do Indo-Hitita}

Por uma inadvertência, deixou-se de juntar a Coorge L. Frazer $\circ$ nome de Henry Lee Smith, Jr., co-autor da colaboração "Uma Cronologia Indo-Hitita". inserto no n. ${ }^{\circ} 3$ de "Letras". 